\title{
Prognostic features for quality of life after radical cystectomy and orthotopic neobladder
}

\author{
Alexander Kretschmer ${ }^{1}$, Tobias Grimm ${ }^{1}$, Alexander Buchner ${ }^{1}$, Christian G. Stief ${ }^{1}$, Alexander Karl ${ }^{1}$ \\ ${ }^{1}$ Department of Urology, Ludwig - Maximilians - University, Munich, Germany
}

\section{ABSTRACT}

Purpose: To analyse prognostic features on quality of life (QoL) following radical cystectomy and urinary diversion via orthotopic neobladder in a single-centre patient cohort.

Materials and Methods: Postoperative QoL of 152 patients was assessed retrospectively using the validated QLQ-C30 questionnaire. Potential associations of patient's quality of life including pre-and intraoperative characteristics, surgeon experience, postoperative time course, adjuvant therapies, and functional outcome were defined a priori and evaluated. Mann-Whitney-U-, Kruskal-Wallis-, Spearman correlation and post hoctesting were used. A multivariate analysis using a multiple logistic regression model was performed. A p value $<0.05$ was considered to be statistically significant.

Results: Median follow-up was 48 months. Univariate analysis of prognostic features for health-related QoL revealed a significant impact of gender $(\mathrm{p}=0.019)$, performance status $(p<0.001)$, experience of surgeon $(>100$ previous cystectomies, $p=0.007)$, and nerve-sparing surgery $(p=0.001)$. Patients who underwent secondary chemotherapy or radiotherapy had significant lower QLQ-C30 scores ( $p=0.04, p=0.02$ respectively). $\mathrm{Pa}$ tients who were asymptomatic had a significantly higher quality of life $(\mathrm{p}<0.001)$. A significant impact of severity of incontinence based on ICIQ-SF score $(p<0.001)$ and daily pad usage $(\mathrm{p}<0.001)$, existence of daytime incontinence $(\mathrm{p}<0.001)$, existence of urgency symptoms $(\mathrm{p}=0.007)$, and IIEF-5 score $(\mathrm{p}<0.001)$ could be observed. In multivariate analysis, independent prognostic relevance could be confirmed for preoperative ECOG performance status of 0 ( $p=0.020$ vs. ECOG $1, p=0.047$ vs. ECOG 2), experience of the respective surgeon ( $\geq 100$ vs. $<100$ previous cystectomies, $p=0.021$ ), and daytime continence $(\mathrm{p}=0.032)$.

Conclusion: In the present study, we report health-related QoL outcomes in a contemporary patient cohort and confirm preoperative ECOG status, surgeon experience and daytime incontinence as independent prognostic features for a good postoperative QoL.

\section{ARTICLE INFO}

\section{Keywords:}

Quality of Life; Cystectomy; Urinary Bladder Neoplasms; Urinary Incontinence

Int Braz J Urol. 2016; 42: 1109-20

Submitted for publication:

September 06, 2015

Accepted after revision:

May 10, 2016

Published as Ahead of Print:

August 11, 2016

\section{INTRODUCTION}

Bladder cancer is the second most common malignancy in the urinary tract and transitional cell carcinoma of the urinary bladder is currently the fourth most common malignancy in men in the Western world (1). Neoadjuvant chemotherapy, followed by radical cystectomy, extended pelvic lymphadenectomy, and urinary diversion (using an ileal conduit or ileal orthotopic neobladder) is the gold standard therapeutic approach for muscle invasive bladder cancer (2). However, radical cystectomy comes along with quite a burden for the patient considering a long-term morbidity-rate of 
up to $90 \%$ leading to a considerable mortality rate (3-5). Recently, there was an increasing interest in QoL aspects for patients undergoing radical cystectomy, mostly regarding different types of urinary diversion (6). While short-term and long-term complications and long-term functional outcomes after urinary diversion via orthotopic neobladder have been extensively analysed there is still a lack of data concerning QoL aspects. Functional outcomes have been described to significantly influence the patient's QoL after orthotopic urinary diversion, but little is known about further possible influencing factors (7). Thus, the aim of this study is to investigate various potential contributing factors on patient's QoL after radical cystectomy and urinary diversion via orthotopic neobladder.

\section{MATERIALS AND METHODS}

Patient population, study design and data assessment

In one tertiary care centre, 301 cystectomies with urinary diversion via orthotopic neobladder were performed between May 2004 and September 2014 by a total number of 14 surgeons. During follow-up period, 92 patients had died. After approval by an institutional review board, data assessment was performed retrospectively via standardized questionnaires that were sent to 209 patients who underwent radical cystectomy and urinary diversion via orthotopic neobladder due to malignancy. Questionnaires were returned by 152 patients and underwent further analysis. This leads to a global response rate of 50.5\%.

Health-related QoL was measured using the standardized EORTC QLQ-C30 (global health status; validated German version) (8). The global health status was calculated following current EORTC instructions: $s c o r e=100^{*}$ [(question29+question30)/2-1]/6 (9). This indicates that higher scores are associated with an increased QoL.

Potential predefined prognostic features of patient's QoL were evaluated. Hereby we focused on various categories including preoperative characteristics (gender, preoperative age, performance status), intraoperative features (surgeon's experience, nerve sparing operation, operation duration, blood loss, blood transfusion, postoperative revision, postoperative complications), TNM classification, functional outcome (continence status, urgency symptoms, sexual function), adjuvant therapies (chemotherapy, radiotherapy), and symptoms (any symptoms, night sweats, weight loss, flank pain).

The preoperative performance status was determined based on the respective Eastern Cooperative Oncology Group performance (ECOG) status score (10).

Daytime continence was determined using the validated International Consultation on Incontinence Questionnaire (ICIQ) short-form scoring system in a validated German translation, and pad usage (11). The existence of urgency symptoms was assessed. Depending on the respective ICIQ-SF, patient's stress urinary incontinence was classified as mild (1-5), moderate (6-10), and severe $(>10)$ as previously described (11).

Sexual function was assessed using the abridged International Index of Erectile Function (IIEF-5) (12) and abridged Female Sexual Function Index (FSFI-6) (13) respectively. A cut-off score of $>20$ (out of 25) points was used as the definition of potency as previously described (12). Female sexual dysfunction was defined by a FSFI- 6 of less than 18 as previously described by Isidori et al. (13).

\section{Statistical analysis}

Primary endpoint was the QoL (global health status based on EORTC QLQ-C30 questions 29 and 30) of the respective patient. Secondary endpoints were above-mentioned prognostic features. Analysis was performed using the global health status as a continuous variable as well as using a cut-off score of 70 as recently described by Snyder et al. (14). Hereby, a QLQ-C30 score of less than 70 is associated with a poor quality of life, a score of at least 70 is considered to be associated with a good quality of life.

To analyse and compare QoL in the respective subgroups, Mann-Whitney-U-, Kruskal-Wallis-ANOVA, and chi-squared-Test, Spearman's rank correlation, and post-hoc-testing were used whenever indicated. Additionally, a multiple lo- 
gistic regression model that included all potential prognostic features that showed significant results in the univariate analysis was performed. All statistical analyses were performed using STATISTICA 10 software (StatSoft, Tulsa, OK, USA). A p value $<0.05$ was considered to be statistically significant.

\section{RESULTS}

Pre-and perioperative patient characteristics Median follow-up, defined as time between radical cystectomy and answering of the questionnaire, was 48 months [3-108]. Follow-up was within the first 12 months in $22 \%(n=33)$ of the patient collective. Median patient age was 71 years [44-88 years]. Patient characteristics of the patients that underwent further analysis are summarized in Table- 1 .

Analysis responder vs. non-responder

To analyse for a potential selection bias, we compared preoperative characteristics and pathological tumour stage for those patients who responded to the questionnaire and those who did not respond. Among the patients who did not respond to the questionnaire, 56\% were deceased. Median

Table 1 - Baseline patients characteristics, histopathologic results, and stated symptoms following radical cystectomy and orthotopic urinary diversion after a median follow-up of $\mathbf{4 8}$ months.

Gender

$\begin{array}{cc}\text { Male } & 87.5 \%(133 / 152) \\ \text { Female } & 12.5 \%(19 / 152)\end{array}$

Age at follow-up (years)

Median

71 (range 44 to 88 )

pT-Stage

$22.4 \%(34 / 152)$

pT1

$23.0 \%(35 / 152)$

pT2

$27.6 \%(42 / 152)$

pT3

pT4

Lymphadenectomy performed

Yes

No

$\mathrm{pN}$-Stage

pNO

$\mathrm{pN}+$

Symptoms at follow-up
$91.4 \%(139 / 152)$

$8.6 \%(13 / 152)$

$89.2 \%(15 / 139)$

$10.8 \%(15 / 139)$

$18.4 \%(28 / 152)$

$4.6 \%(7 / 152)$

$34.2 \%(50 / 146)$

$65.8 \%(96 / 146)$

$3.9 \%(6 / 152)$

$15.1 \%(22 / 146)$

$6.2 \%(9 / 146)$

$4.1 \%(6 / 146)$

$8.9 \%(13 / 146)$ 
age was 65 years for both groups $(p=0.400)$. Regarding the non-responder group, $48 \%$ had a locally advanced disease (pT3-4) (responder: 25\%, $\mathrm{p}<0.001$ ), and $30 \%$ had lymph node infiltration during cystectomy (responder: 12\%, p<0.001). There were no significant differences regarding the ratio of high-grade tumours (non-responder: 90\% vs. responder: $86 \%, \mathrm{p}=0.359)$, metastatic disease ( $9 \%$ vs. $9 \%, p=0.971)$, and (concomitant) carcinoma in situ (39\% vs. $48 \%, p=0.108$ ).

\section{Functional outcome}

Median ICIQ-SF within our patient collective was 10 (1-21). Depending on the respective ICIQ-SF scores, incontinence was classified as severe in $44 \%(n=67)$ of our study group. Regarding pad usage, $40 \%(n=61)$ were in need of more than 2 pads daily. Incontinence results are summarized in Table-2.

Data concerning sexual function was available for 126 out of 133 male patients and for 15 out of 19 female patients. Analysis of the respective IIEF-5 scores showed a median IIEF-5 of 3 (1-24) within our study collective. Regarding the female patients, median FSFI-6 was 4 (1-20). Sexual function results are summarized in Table- 2 .

Univariate analysis of prognostic features for health-related QoL

\section{Preoperative characteristics}

At median follow-up, overall health-related QLQ-C30 was 75 for male patients and 67 for female patients $(p=0.019)$. Patients with less or equal 71 years (median age of the patient collective) had a median QLQ-C30 of 69, whereas patients older than 71 years had a median score of $74(p=0.784)$. Analysing patient's age continuously using Spearman's correlation, we found no statistically significant impact on the respective QoL $(\mathrm{p}=0.926)$. Regarding the preoperative performance status, we found patients with a preoperative ECOG score of 0 having a significantly higher QoL compared to patients with a preoperative ECOG score of 1 or 2 ( $<<0.001$ respectively, Figure-1A).

Intraoperative characteristics

QoL for patients whose cystectomy was performed by an experienced surgeon ( $>100$ previous

Table 2 - Functional outcome following radical cystectomy and orthotopic urinary diversion after a median follow-up of 48 months.

ICIQ-SF at follow-up

Median

Severity of incontinence based on ICIQ-SF

Pad usage at follow-up

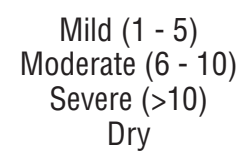

$0-1$

2

IIEF-5 at follow-up

$$
\begin{aligned}
& \text { Median } \\
& \text { Unknown } \\
& >20 \\
& \leq 20
\end{aligned}
$$

FSFI-6 at follow-up
10 (range 4 to 21)

$16.4 \%(25 / 152)$

$37.5 \%(57 / 152)$

$44.1 \%(67 / 152)$

$2.0 \%(3 / 152)$

$37.5 \%(57 / 152)$

$22.3 \%(34 / 152)$

$40.1 \%(61 / 152)$

3 (range 1 to 24)

$5.2 \%(7 / 133)$

$5.6 \%(7 / 126)$

$94.4 \%(119 / 126)$

$$
\begin{aligned}
& \text { Median } \\
& \text { Unknown } \\
& >18 \\
& <18
\end{aligned}
$$

4 (range 1 to 20)

$21,1 \%(4 / 19)$

$13,3 \%(2 / 15)$

$86.7 \%(13 / 15)$

ICIQ-SF = International Consultation on Incontinence Questionnaire short-form; IIEF-5 = International Index of Erectile Function; FSFI-6 = abridged Female Sexual Function Index 
radical cystectomies) had a median QLQ-C30 score of 83 whereas patients whose radical cystectomies were performed by a less experienced surgeon ( $<100$ previous radical cystectomies) had a median QLQ-C30 score of 74 ( $p=0.186$, Figure-1B). However, when using the predefined cut-off value, 53\% (experienced) vs. 35\% (less experienced) of the patients had a QLQ-C30 score of $>70(\mathrm{p}=0.019)$.

A nerve-sparing radical cystectomy was performed in 77 of 152 patients (50.7\%). If a nerve-sparing operation was performed, median postoperative QoL was significantly increased (QLQ-C30 82 vs. 69; $p=0.001$, Figure-1C). 64\% of the nerve-sparing group had a QLQ-C30>70 (vs. $36 \%, \mathrm{p}<0.001)$.

There were no statistical significances regarding intraoperative complications (44 vs. 50\% QLQ-C30 $\geq 70, \mathrm{p}=0.731$ ), postoperative complications (49 vs. 51\%, $p=0.744$ ), need for perioperative blood transfusion ( 36 vs. 53\%, $\mathrm{p}=0.116$ ), intraoperative blood loss $(<500 \mathrm{~mL}$ : 55\% QLQ-C30 $\geq 70$ vs. $\geq 500 \mathrm{~mL}: 47 \%, p=0.355$ ), and the operation duration (median operation time: 217 minutes; $<217$ minutes: 58\% QLQ-C30 $\geq 70$ vs. 43\%, $\mathrm{p}=0.077$ ).

Regarding the pathological tumour stage, patients with locally advanced disease (pT3-4) as well as patients with lymph node involvement did not have statistically significant different QLQ-C30 scores than those with limited disease as well as without lymph node involvement $(\mathrm{p}=0.760$, $\mathrm{p}=0.632$ respectively).

\section{Postoperative time course and adjuvant the-} rapies

Regarding the postoperative time course, we found no statistically significant differences when comparing patient's QOL after 3 months, 6 months, 9 to 12 months, 1 year, 2 to 3 years, 4 to 5 years, and more than 5 years postoperatively. In detail, patients who were analysed during their first year of follow-up had a median QLQ-C30 of 67 (39\% QLQ-C30 $\geq 70)$; patients who were analysed after more than one year postoperatively had a median score of $75[\mathrm{p}=0.078$ (53\% QLQ-C30 $\geq 70, p=0.168)]$.

Adjuvant systemic chemotherapy or palliative chemotherapy due to tumour recurrence was performed in 19 of 152 patients (12.5\%). Median
QLQ-C30 score for those patients was 67 (vs. 82 if no chemotherapy was performed, $p=0.04$ ). Among the patients who underwent chemotherapy, 33\% had a QLQ-C30 of $\geq 70$ (vs. 52\%, $p=0.174$ ). There was no significant difference if the chemotherapy was performed within the last 12 months before answering the questionnaire, or more than 12 months ago ( $p=0.635)$.

During the postoperative time course, radiotherapy was performed in 5 of 152 patients (3.9\%). Median QLQ-C30 was 77 if no radiotherapy was performed, and 41 if radiotherapy was performed $(p=0.006)$. None of the patients who underwent radiotherapy reached a QLQ-C30 score of $\geq 70$ ( $p=0.02$ ).

In total, 19 patients (12.5\%) suffered from recurrence of their malignancy. There was no statistically significant difference when comparing these patients with those patients without recurring disease (41\% QLQ-C30 $\geq 70$ vs. 52\%, $\mathrm{p}=0.462$ ).

Patients who were symptomatic at the time of follow-up had a significantly lower QoL than those being asymptomatic $(p=0.006$, Figure-1D). In detail, patients suffering from weight loss had a significantly lower QoL than asymptomatic ones $(p=0.003)$ whereas patients with night sweats as well as flank pain did not ( $p=0.076, p=0.214$ respectively). The symptomatic as well as asymptomatic patients did not differ in terms of disease extent and lymph node involvement $(p=0.800)$. Among the asymptomatic patients, 60\% had a QLQ-C30 of $\geq 70$ (symptomatic patients: $34 \%, p=0.003$ ).

\section{Functional outcome}

Addressing the potential impact of incontinence, we found a median QLQ-C30 of 69 for daytime incontinence and a median QLQ-C30 of 82 for daytime continence $(p<0.001$, Figure-1E). Impact of severity of incontinence was analysed based on daily pad usage [0-1 pad (83) vs. $>2$ pads (65), $p<0.001$, Figure-1F], and based on the ICIQ-SF [dry/mild incontinence (median QLQ-C30: 83) vs. moderate incontinence (83) vs. severe incontinence (67), $\mathrm{p}<0.001$, Figure-1G]. Continuous analysis of the respective ICIQ-SF scores confirmed our primary results ( $>0.001$, Figure-2). Among the dry patients as well as patients with mild incontinen- 
Figures $1 \mathrm{~A}-\mathrm{H}$ - Univariate analysis of multiple predefined prognostic features on postoperative QoL after radical cystectomy and orthotopic urinary diversion.
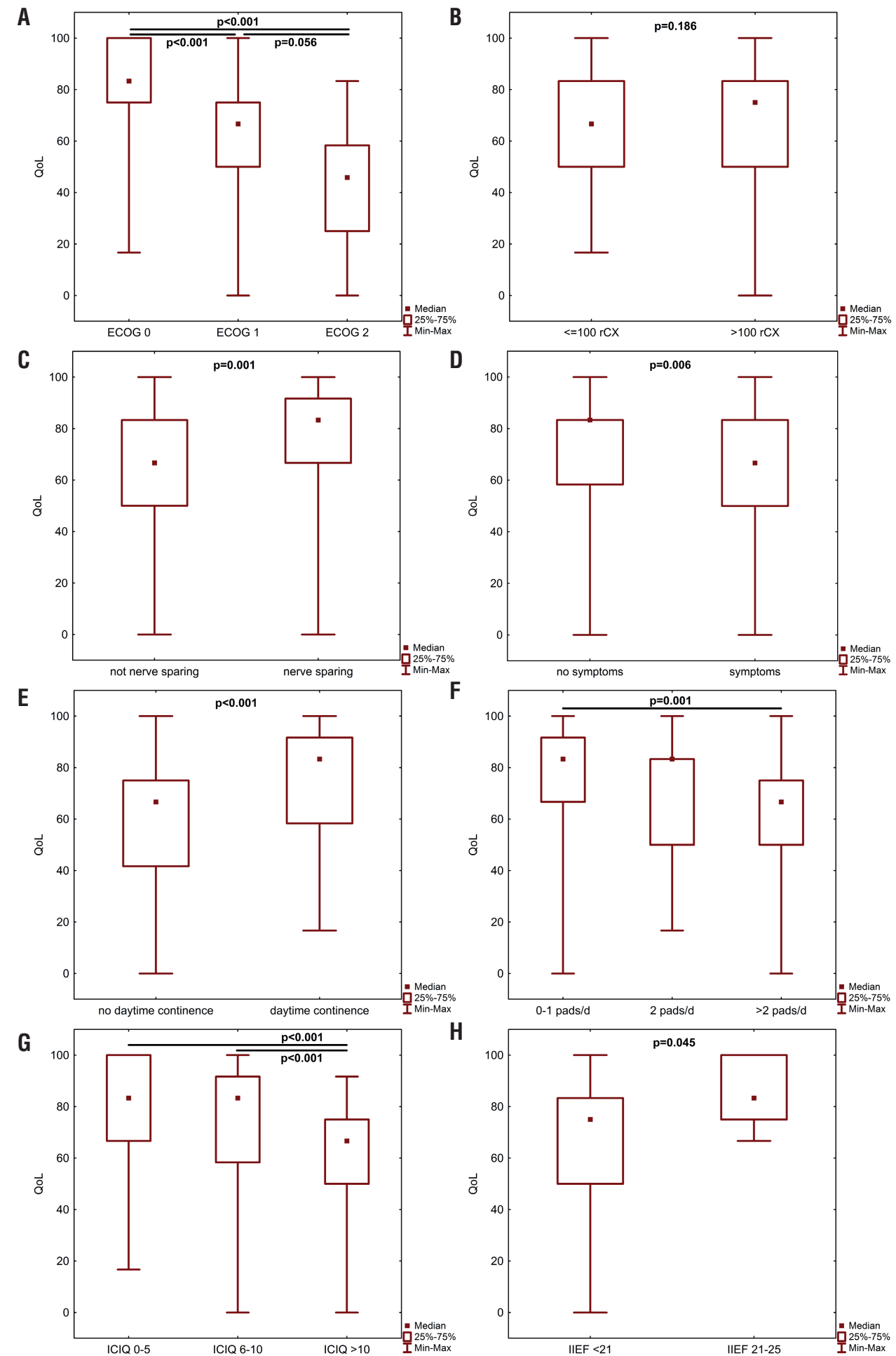

A p value $<0.05$ was considered statistically significant $(\mathbf{E C O G}=$ Eastern Cooperative Oncology Group performance status score; ICIQ-SF=International Consultation on Incontinence Questionnaire short-form; IIEF-5=International Index of Erectile Function; $\mathbf{Q} \mathbf{0 L}=q$ quality of life; $\mathbf{r C} \mathbf{x}=$ radical cystectomy). 
Figure 2 - Impact of patient's ICIQ-SF score on health-related quality of life based on EORTC QLQ-C30 global health status using Spearman's rank correlation. A p value $<0.05$ was considered statistically significant (QOL=quality of life).

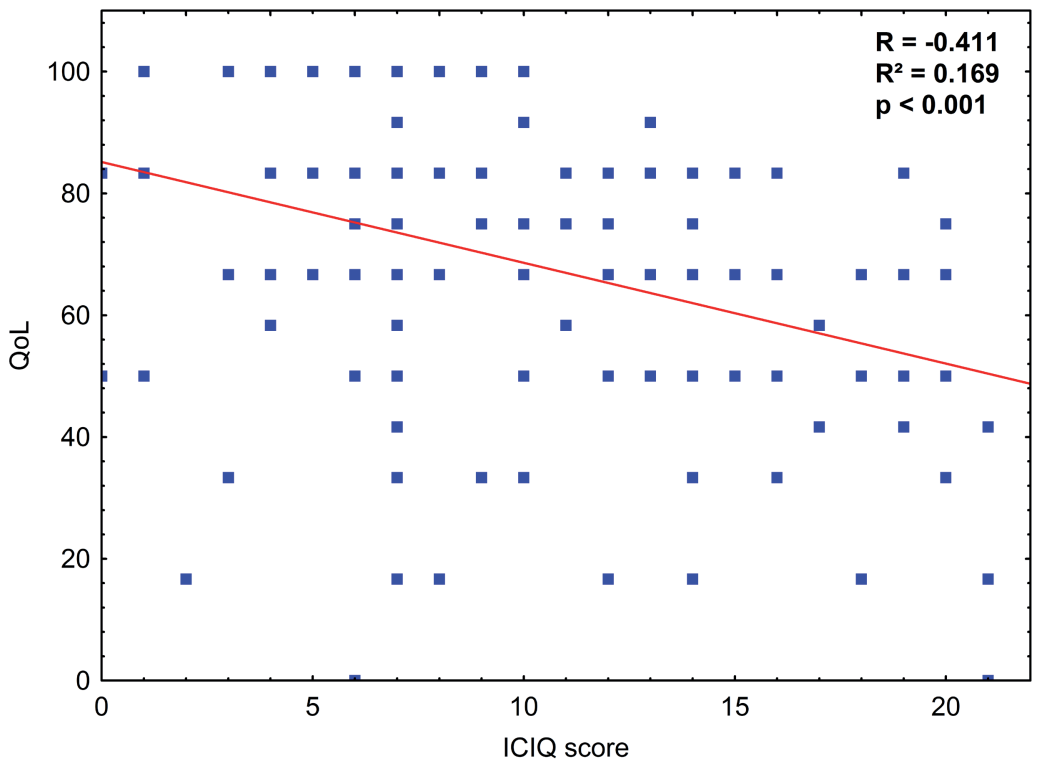

ce, 69\% had a QLQ-C30 score of $\geq 70$ (moderate incontinence: 66\%, severe incontinence: $28 \%$; $\mathrm{p}<0.001)$. In line, $65 \%$ of the patients in need of $0-1$ pads per $24 \mathrm{~h}$ had a QLQ-C30 score of $\geq 70$ (2 pads: $62 \%,>2$ pads: $30 \% ; \mathrm{p}<0.001)$. Among the patients that achieved daytime continence, $61 \%$ had a QLQ-C30 score of $\geq 70$ (no daytime continence: $28 \%, \mathrm{p}<0.001)$. Patients suffering from urinary urgency had a median QLQ-C30 of 70, whereas patients with no urgency symptoms had a median score of $82(p=0.015)$. Regarding the predefined cut-off values, $28 \%$ of the patients suffering from urinary urgency achieved a QLQ-C30 score of $\geq 70$ (no urgency symptoms: 55\%, $\mathrm{p}=0.007$ ).

Analysis of sexual outcome revealed a significant influence of IIEF-5 $[<20$ (median QLQ-C30: 75) vs. $>20$ (83), $\mathrm{p}=0.045$, Figure$-1 \mathrm{H}$ ], but not of FSFI-6 (FSFI- $6 \leq 18$ vs. FSFI $>18$; $\mathrm{p}=0.800$ ). The patient subgroup that skipped the IIEF-5 questionnaire did not differ statistically significant in age $(\mathrm{p}=0.786)$ as well as ICIQ-SF $(p=0.863)$ to those who answered the questionnaire. Among the patients with an IIEF-5 score of 20-25, 86\% achieved a QLQ-C30 score of $\geq 70$ (<20: 51\%, p=0.078).
Multivariate analysis of prognostic features for health-related QoL

In a last step, a multivariate analysis was performed using a multiple logistic regression model. Hereby, all prognostic features that had statistically significant results in the univariate analysis were included. In detail, independent prognostic relevance could be confirmed for preoperative ECOG performance status of 0 ( $p=0.020$ vs. ECOG $1, p=0.047$ vs. ECOG 2), the experience of the respective surgeon ( $\geq 100$ vs. $<100$ previous cystectomies, $\mathrm{p}=0.021)$, and daytime continence $(\mathrm{p}=0.032)$. Results of the multivariate analysis are summarized in Table-3.

\section{DISCUSSION}

Orthotopic ileal neobladder has become a widespread treatment of muscle-invasive bladder cancer. Certainly, complication rates are still high and functional outcomes may be unfavourable even in high-volume centres (15-18). As a consequence, recently more attention was drawn towards health-related QoL after radical cystectomy (6). However, measurement of QoL remains difficult and requires specific tools to address 
Table 3 - Multivariate analysis of prognostic features that showed significant results in univariate analysis.

\begin{tabular}{lcccc}
\hline Prognostic feature & P value & OR & \multicolumn{2}{c}{$95 \% \mathrm{Cl}$} \\
\hline Gender [male/female] & 0.185 & 2.745 & 0.618 & 12.193 \\
ECOG 1 vs. 0 & $\mathbf{0 . 0 2 0}$ & 0.336 & 0.134 & 0.842 \\
ECOG 2 vs. 0 & $\mathbf{0 . 0 4 7}$ & 0.121 & 0.015 & 0.974 \\
ICIQ group mild vs. moderate & 0.394 & 1.76 & 0.48 & 6.449 \\
ICIQ group mild vs. severe & 0.382 & 0.504 & 0.109 & 2.342 \\
Surgeon experience $<100$ vs. $>100$ prev. rCx [y/n] & $\mathbf{0 . 0 2 1}$ & 3.442 & 1.203 & 9.847 \\
Nerve-sparing [y/n] & 0.165 & 2.029 & 0.747 & 5.511 \\
Radiation therapy [y/n] & 0.999 & 0 & 0 &. \\
Symptoms [y/n] & 0.109 & 0.458 & 0.176 & 1.192 \\
Urge [y/n] & 0.259 & 0.486 & 0.139 & 1.702 \\
Daytime continence [y/n] & $\mathbf{0 . 0 3 2}$ & 3.131 & 1.101 & 8.908 \\
Daily pad usage 2 vs. $0-1$ & 0.817 & 0.859 & 0.238 & 3.105 \\
Daily pad usage $>2$ vs. $0-1$ & 0.995 & 0.996 & 0.274 & 3.616 \\
\hline
\end{tabular}

$\mathbf{C I}=$ confidence interval; $\mathbf{E C O G}$ = Eastern Cooperative Oncology Group performance status score; $\mathbf{I C I} \mathbf{Q}=$ International Consultation on Incontinence Questionnaire; $\mathbf{O R}=$ odds ratio; $\mathbf{r} \mathbf{C} \mathbf{x}=$ radical cystectomy.

A p value $>0.05$ was considered statistically significant.

health-related QoL aspects adequately. A various number of different existing instruments-generic, cancer specific and bladder cancer specific-complicate the comparison and integration of the pre-existing literature (19). We used the validated EORTC QLQ-C30 that has been frequently used in multiple studies investigating QoL after radical cystectomy (20-27). In recent years, many efforts have been made to define certain cut-off values in order to select those patients with unmet needs for further support. Snyder et al. evaluated more than 500 oncologic patients and found a QLQ-C30 global health status cut-off value of 70 to have a sensitivity of up to $86 \%$ and a negative predictive value of up to $94 \%(14,28)$. The aim of the current study was to evaluate various potential prognostic features on patients QoL after orthotopic urinary diversion, focusing on preoperative, perioperative as well as postoperative features. To allow a better comparison with existing literature, we analysed the postoperative QLQ-C30 global health status as a continuous parameter as well as following Snyder et al. (14).

Addressing patient-derived features, we found no statistically significant impact of preoperative patient's age. This funding is in line with recent publications indicating that radical cystectomy with orthotopic urinary diversion can be safely performed even in elderly patient cohorts (29). Imbimbo et al. even found significant increased QLQ-C30 scores in patients older than 65 years (30). However, there is also evidence that elderly patients are still less likely to receive orthotopic urinary diversion (31). Going further, we found a significant impact of the preoperative performance status on postoperative QoL in univariate as well as in multivariate analysis. Given the fact that health-related QoL measurements take into consideration multiple aspects including physical status, general health and social interaction, it seems intuitive that patients with a better performance status profit more from a procedure that aims to allow an unaltered, active lifestyle. In line, it has been shown that patients undergoing orthotopic urinary diversion are more active and have a better physical function compared to patients undergoing incontinent urinary diversion (32). Our observations indicate that age per se is not associated with impaired QoL after orthotopic urinary diversion and discussion of QoL aspects prior to radical cystectomy should 
be based on the patient's performance status rather than on the respective patient's age. Regarding preoperative patient selection, our results indicate, that elderly patients who have an adequate performance status, can be treated with an orthotopic urinary diversion and still have a good postoperative QoL. Since radical cystectomy for octogenarians becomes more accepted, this finding has important clinical implications (33).

In the current study, various intraoperative characteristics on the patient's postoperative QoL were analysed, indicating a significant impact of the surgeon's experience both in univariate as well as in multivariate analysis. To our knowledge, evidence regarding the surgeon's learning curve impact on patients postoperative QoL after radical cystectomy and orthotopic urinary diversion is still very limited. However, there is evidence that excellent functional outcomes can be achieved in high-volume centers (18). In line, it has been shown that the surgeon's individual learning curve has a significant impact on functional outcome after radical prostatectomy and is therefore directly affecting the patient's postoperative QoL (34). The impact of surgeon's experience on postoperative QoL after orthotopic urinary diversion might highlight the importance of the referral to high-volume centers and therefore has clinical implications.

Addressing a potential time course of patient's QoL after orthotopic urinary diversion, we found no statistically significant differences in QoL after the respective moments of follow-up. However, there was a statistical trend favouring those patients who were evaluated more than 1 year postoperatively. This might indicate that QoL increases during the postoperative course of time as described by Kulaksizoglu and colleagues (27). We found that, if patients underwent adjuvant or palliative chemotherapy or radiotherapy, QoL was significantly lower, no matter if the respective therapy was performed within 1 year before answering the questionnaires or not. It has to be stated, however, that there might be a relevant non-responder bias in our patient cohort. Thus, these findings have to be interpreted with caution. Our results are partly in line with the findings of Cognetti et al. who also found a decline of QoL after initiation of adjuvant Cisplatin-based chemotherapy. However, the authors stated that there was a subsequent improvement after the initial decline of QoL, which is not reflected by our data (35).

To assess the impact of urinary incontinence on health-related QoL, we used the validated ICIQ-SF questionnaire and pad usage and found a significant impact of urinary leakage. These findings are in line with the results of Takenaka et al. who evaluated long-term outcome in QoL after orthotopic neobladder in 86 patients and found worse QoL scores in patients with daytime incontinence (36). Zahran et al. analyzed QoL of 74 women after radical cystectomy and orthotopic neobladder using QLQ-C30 and observed a significant negative impact of incontinence (7). In line with Imbimbo et al., we found urinary incontinence being an independent prognostic feature of an impaired QoL (30). However, the findings of the current study addressing the great impact of continence are based on multiple consistent parameters in a contemporary patient collective and therefore add important information to the existing literature.

It is commonly accepted that a significant proportion of patients after orthotopic bladder replacement suffer from sexual dysfunction (37). In our patient collective, for instance, only $5.6 \%$ of the men could be classified as "no sexual dysfunction" based on the respective IIEF-5 score (>20). Since no preoperative data was available, these results have to be interpreted with caution. However, we were able to show that health-related QoL was significantly increased in those patients who had a postoperative IIEF-5 score of more than 20 . To date, there is only limited data concerning the impact of sexual function on QoL after radical cystectomy. Studies from large prostate cancer series, on the other hand, consistently report better QoL outcomes for nerve-sparing radical prostatectomies indicating a major impact of sexual preservation on QoL aspects after major pelvic surgery (38). Summarizing, our findings highlight the great impact of functional aspects on health-related QoL. However, it is somewhat difficult to imply the important role that postoperative continence plays for health-related 
QoL into daily clinical practice. Naturally, every surgeon tries to achieve the best possible functional result. However, one might focus on the development of continence-preserving technical modifications. Regarding radical prostatectomy for example, the posterior rhabdomyosphincter reconstruction, also known as Rocco stitch, has been proposed to improve postoperative continence $(39,40)$. Additionally, we still lack knowledge how the shape of the neobladder (e.g. U- vs. W-shape) affects postoperative continence recovery.

Certainly, our study is not devoid of limitations. Major drawback is its retrospective nature with all the known limitations that are inherent. The current study is a cross-sectional study and QoL has not been measured at specific time-points. Nevertheless, the current study provides QoL analyses from various different follow-up periods, providing a possible idea of the natural process of QoL after radical cystectomy. In the current study, the EORTC QLQ-C30 global health status was used. It has to be stated, that, unlike the EORTC QLQ-BLM30, this questionnaire is not bladder cancer specific and lacks additional information regarding body image, urostomy problems, and use of catheters. The QLQ-BLM30 has been used in several publications before, but is still not validated (23, 26, 41). Additionally, the Functional Assessment of Cancer Therapy-General, Bladder (FACT-BL) and Vanderbilt Cystectomy Index (FACT-VCI) are validated bladder cancer specific questionnaires that have been used in health-related QoL studies before (42). However, in a recent meta-analysis including data from 18 studies that investigated health-related QoL after radical cystectomy, 10 out of 18 used the EORTC QLQ-C30 questionnaire (43). This indicates, that, despite being not cancer specific, the QLQ-C30 questionnaire is well established in QoL analyses of patient cohorts that underwent radical cystectomy and orthotopic urinary diversion.

\section{CONCLUSIONS}

The current study analyses the impact of multiple potentially prognostic features on QoL after orthotopic urinary diversion. We report health-related QoL outcomes in a contemporary patient cohort. Hereby, we were able to show that preoperative ECOG status, surgeon experience and daytime incontinence are independent prognostic features for a good postoperative QoL.

\section{ABBREVIATIONS}

QoL $=$ Quality of life

QLQ-C30 = Quality of life questionnaire C30

EORTC = European Organization for Research on Treatment of Cancer

ICIQ-SF = International Consultation on Incontinence Questionnaire short-form

IIEF-5 = International Index of Erectile Function

FSFI-6 = Female Sexual Function Index (abridged)

FACT-BL $=$ Functional Assessment of Cancer Therapy-General, Bladder

FACT-VCI = Vanderbilt Cystectomy Index

\section{CONFLICT OF INTEREST}

None declared.

\section{REFERENCES}

1. Siegel R, Ma J, Zou Z, Jemal A. Cancer statistics, 2014. CA Cancer J Clin. 2014;64:9-29. Erratum in: CA Cancer J Clin. 2014;64:364.

2. Witjes JA, Compérat E, Cowan NC, De Santis M, Gakis $G$, Lebret $T$, et al. EAU guidelines on muscle-invasive and metastatic bladder cancer: summary of the 2013 guidelines. Eur Urol. 2014;65:778-92.

3. Hautmann RE, Hautmann SH, Hautmann 0. Complications associated with urinary diversion. Nat Rev Urol. 2011;8:667-77.

4. Nam JK, Kim TN, Park SW, Lee SD, Chung MK. The Studer orthotopic neobladder: long-term (more than 10 years) functional outcomes, urodynamic features, and complications. Yonsei Med J. 2013;54:690-5.

5. Raza SJ, Ather MH, Khan FA, Alam Z. Grading complication following radical cystectomy and ileal conduit for bladder cancer using Clavien grading system. J Coll Physicians Surg Pak. 2012;22:448-51.

6. Ali AS, Hayes MC, Birch B, Dudderidge T, Somani BK. Health related quality of life (HRQLL) after cystectomy: comparison between orthotopic neobladder and ileal conduit diversion. Eur J Surg Oncol. 2015;41:295-9. 
7. Zahran MH, El-Hefnawy AS, Zidan EM, El-Bilsha MA, Taha $\mathrm{DE}$, Ali-El-Dein B. Health-related quality of life after radical cystectomy and neobladder reconstruction in women: impact of voiding and continence status. Int J Urol. 2014;21:887-92.

8. Aaronson NK, Ahmedzai S, Bergman B, Bullinger M, Cull A, Duez NJ, et al. The European Organization for Research and Treatment of Cancer QLQ-C30: a quality-of-life instrument for use in international clinical trials in oncology. J Natl Cancer Inst. 1993;85:365-76.

9. Fayers PM. Interpreting quality of life data: populationbased reference data for the EORTC QLQ-C30. Eur J Cancer. 2001;37:1331-4.

10. Oken MM, Creech RH, Tormey DC, Horton J, Davis TE, McFadden ET, et al. Toxicity and response criteria of the Eastern Cooperative Oncology Group. Am J Clin Oncol. 1982;5:649-55.

11. Avery K, Donovan J, Peters TJ, Shaw C, Gotoh M, Abrams P. ICIQ: a brief and robust measure for evaluating the symptoms and impact of urinary incontinence. Neurourol Urodyn. 2004;23:322-30.

12. Rosen RC, Cappelleri JC, Smith MD, Lipsky J, Peña BM. Development and evaluation of an abridged, 5-item version of the International Index of Erectile Function (IIEF-5) as a diagnostic tool for erectile dysfunction. Int J Impot Res. 1999;11:319-26.

13. Isidori AM, Pozza C, Esposito K, Giugliano D, Morano S, Vignozzi L, et al. Development and validation of a 6 -item version of the female sexual function index (FSFI) as a diagnostic tool for female sexual dysfunction. J Sex Med. 2010;7:1139-46.

14. Snyder CF, Blackford AL, Okuyama T, Akechi T, Yamashita $\mathrm{H}$, Toyama $\mathrm{T}$, et al. Using the EORTC-QLQ-C30 in clinical practice for patient management: identifying scores requiring a clinician's attention. Qual Life Res. 2013;22:2685-91.

15. Nieuwenhuijzen JA, de Vries RR, Bex A, van der Poel HG, Meinhardt W, Antonini N, et al. Urinary diversions after cystectomy: the association of clinical factors, complications and functional results of four different diversions. Eur Urol. 2008;53:834-42.

16. Jentzmik F, Schostak M, Stephan C, Baumunk D, Lingnau $A$, Weikert $S$, et al. Extraperitoneal radical cystectomy with extraperitonealization of the ileal neobladder: a comparison to the transperitoneal technique. World J Urol. 2010;28:457-63.

17. Shabsigh A, Korets R, Vora KC, Brooks CM, Cronin AM, Savage C, et al. Defining early morbidity of radical cystectomy for patients with bladder cancer using a standardized reporting methodology. Eur Urol. 2009;55:164-74.

18. Todenhöfer T, Stenzl A, Schwentner C. Optimal use and outcomes of orthotopic neobladder reconstruction in men and women. Curr Opin Urol. 2013;23:479-86.

19. Dombo 0, Otto U. [Quality of life after radical urologic pelvic surgery and impact of inpatient rehabilitation]. Urologe A. 2005;44:11-28.
20. Kitamura H, Miyao N, Yanase M, Masumori N, Matsukawa $\mathrm{M}$, Takahashi A, et al. Quality of life in patients having an ileal conduit, continente reservoir or orthotopic neobladder after cystectomy for bladder carcinoma. Int J Urol. 1999;6:393-9.

21. Somani BK, Gimlin D, Fayers P, N'dow J. Quality of life and body image for bladder cancer patients undergoing radical cystectomy and urinary diversion - a prospective cohort study with a systematic review of literature. Urology. 2009;74:1138-43.

22. Hobisch A, Tosun K, Kinzl J, Kemmler G, Bartsch G, Höltl $L$, et al. Quality of life after cystectomy and orthotopic neobladder versus ileal conduit urinary diversion. World $\mathrm{J}$ Urol. 2000;18:338-44.

23. Erber B, Schrader M, Miller K, Schostak M, Baumunk $D$, Lingnau $A$, et al. Morbidity and Quality of Life in Bladder Cancer Patients following Cystectomy and Urinary Diversion: A Single-Institution Comparison of Ileal Conduit versus Orthotopic Neobladder. ISRN Urol. 2012;2012:342796.

24. Protogerou V, Moschou M, Antoniou N, Varkarakis J, Bamias A, Deliveliotis C. Modified S-pouch neobladder vs ileal conduit and a matched control population: a quality-of-life survey. BJU Int. 2004;94:350-4.

25. Saika T, Arata R, Tsushima T, Nasu Y, Suyama B, Takeda K, et al. Health-related quality of life after radical cystectomy for bladder cancer in elderly patients with an ileal conduit, ureterocutaneostomy, or orthotopic urinary reservoir: a comparative questionnaire survey. Acta Med Okayama. 2007;61:199-203.

26. Sogni F, Brausi M, Frea B, Martinengo C, Faggiano F, Tizzani $A$, et al. Morbidity and quality of life in elderly patients receiving ileal conduit or orthotopic neobladder after radical cystectomy for invasive bladder cancer. Urology. 2008;71:919-23.

27. Kulaksizoglu H, Toktas G, Kulaksizoglu IB, Aglamis E, Unlüer E. When should quality of life be measured after radical cystectomy? Eur Urol. 2002;42:350-5.

28. Snyder CF, Blackford AL, Brahmer JR, Carducci MA, Pili R, Stearns $V$, et al. Needs assessments can identify scores on HRQOL questionnaires that represent problems for patients: an illustration with the Supportive Care Needs Survey and the QLQ-C30. Qual Life Res. 2010;19:837-45.

29. Bassett JC, Chang SS. Treating octogenarians with muscleinvasive bladder cancer: preoperative opportunities for increasing the benefits of surgical intervention. Urol Oncol. 2014;32:37.e13-6.

30. Imbimbo C, Mirone V, Siracusano S, Niero M, Cerruto MA, Lonardi C, et al. Quality of Life Assessment With Orthotopic Ileal Neobladder Reconstruction After Radical Cystectomy: Results From a Prospective Italian Multicenter Observational Study. Urology. 2015;86:974-9. 
31. Hounsome LS, Abel GA, Verne J, Neal DE, Lyratzopoulos G. Predictors of the use of orthotopic bladder reconstruction after radical cystectomy for bladder cancer: data from a pilot study of 1756 cases 2004-2011. BJU Int. 2013;111:1061-7.

32. Philip J, Manikandan R, Venugopal S, Desouza J, Javlé PM. Orthotopic neobladder versus ileal conduit urinary diversion after cystectomy-a quality-of-life based comparison. Ann R Coll Surg Engl. 2009;91:565-9.

33. Izquierdo L, Peri L, Leon P, Ramírez-Backhaus M, Manning T, Alcaraz A, et al. The role of cystectomy in elderly patients - a multicentre analysis. BJU Int. 2015;116:73-9. Erratum in: BJU Int. 2016;117:88.

34. Kretschmer A, Mandel P, Buchner A, Stief CG, Tilki D. Surgical learning curve for open radical prostatectomy: Is there an end to the learning curve? World $\mathrm{J}$ Urol. 2015;33:1721-7.

35. Cognetti F, Ruggeri EM, Felici A, Gallucci M, Muto G, Pollera CF, et al. Adjuvant chemotherapy with cisplatin and gemcitabine versus chemotherapy at relapse in patients with muscle-invasive bladder cancer submitted to radical cystectomy: an Italian, multicenter, randomized phase III trial. Ann Oncol. 2012;23:695-700.

36. Takenaka A, Hara I, Soga H, Sakai I, Terakawa T, Muramaki $M$, et al. Assessment of long-term quality of life in patients with orthotopic neobladder followed for more than 5 years. Int Urol Nephrol. 2011;43:749-54.

37. Novara G, Ficarra V, Minja A, De Marco V, Artibani W. Functional results following vescica ileale Padovana (VIP) neobladder: midterm follow-up analysis with validated questionnaires. Eur Urol. 2010;57:1045-51.

38. Punnen S, Cowan JE, Chan JM, Carroll PR, Cooperberg MR. Long-term health-related quality of life after primary treatment for localized prostate cancer: results from the CaPSURE registry. Eur Urol. 2015;68:600-8.
39. Rocco B, Gregori A, Stener S, Santoro L, Bozzola A, Galli $S$, et al. Posterior reconstruction of the rhabdosphincter allows a rapid recovery of continence after transperitoneal videolaparoscopic radical prostatectomy. Eur Urol. 2007;51:996-1003.

40. Rocco F, Carmignani L, Acquati P, Gadda F, Dell'Orto P, Rocco $B$, et al. Early continence recovery after open radical prostatectomy with restoration of the posterior aspect of the rhabdosphincter. Eur Urol. 2007;52:376-83.

41. Gacci M, Saleh O, Cai T, Gore JL, D'Elia C, Minervini A, et al. Quality of life in women undergoing urinary diversion for bladder cancer: results of a multicenter study among longterm disease-free survivors. Health Qual Life Outcomes. 2013;11:43.

42. Cookson MS, Dutta SC, Chang SS, Clark T, Smith JA Jr, Wells $N$. Health related quality of life in patients treated with radical cystectomy and urinary diversion for urothelial carcinoma of the bladder: development and validation of a new disease specific questionnaire. J Urol. 2003;170:1926-30.

43. Cerruto MA, D'Elia C, Siracusano S, Gedeshi X, Mariotto $A$, lafrate $M$, et al. Systematic review and meta-analysis of non RCT's on health related quality of life after radical cystectomy using validated questionnaires: Better results with orthotopic neobladder versus ileal conduit. Eur J Surg Oncol. 2016;42:343-60.

\footnotetext{
Correspondence address: Alexander Kretschmer, MD Department of Urology Ludwig-Maximilians - University Marchioninistrasse 15, 81377, Munich, Germany Fax: +49 894 4007-5444
}

E-mail: alexander.kretschmer@med.uni-muenchen.de 